\title{
Functional variability in movement coordination and perception
}

\author{
RICHARD E. A. VAN EMMERIK | JOSEPH HAMILL' ${ }^{1}$ JEFFREY M. HADDAD² \\ 1 Department of Kinesiology, University of Massachusetts Amherst, USA. \\ 2 Department of Health and Kinesiology, Purdue University, USA. \\ Correspondence to: Richard E.A. van Emmerik. Department of Kinesiology, University of Massachusetts Amherst, 30 Eastman Lane, Amherst, MA 01003. \\ Phone: +1-413-545-0325 \\ email: rvanemmerik@umass.edu \\ https://doi.org/10.20338/bjmb.v15i5.248
}

ABBREVIATIONS
UCM Uncontrolled manifold

PUBLICATION DATA

Received 07062021

Accepted 10072021

Published 01122021

\section{INTRODUCTION}

Over the last decades significant advances have been made in our understanding about the functional role of variability in human movement. These advances reflect a shift from perspectives that emphasized variability as noise and detrimental to being functional and essential in regard to adaptation, exploration and learning. ${ }^{1}$ In the dynamical systems perspective variability is an essential aspect of adaptive movement and represents a key variable in the identification and emergence of transitions in movement coordination and the loss of functionality due to movement disability and injury..$^{2-4}$

Another key development in our changing perspective on the role of variability in human movement is the recognition that movements are not only performatory but also are essential in the process of exploration and the pick-up of information about oneself and the environment. ${ }^{5}$ From this perspective, variability may not serve immediate control objectives but provides task-relevant information about the interaction between the organism and its environment. In the area of human postural control this is directly linked to the concept of stability limits (such as the boundary of the base of support provided by the feet) for upright stance and how postural control emerges in relation to those boundaries. From this ecological perspective, stability of stance is more closely related to the proximity of the foot center of pressure or body center of mass to those boundaries than the amount of movement in these variables per se..$^{5-7}$ Assessing variability of postural control with or without incorporating these boundaries can lead to different interpretations of functional declines due to aging and disease as it allows assessments of loss of sensitivity to these boundaries.

Several aspects need to be addressed that will further develop our understanding of the role of variability in human movement. A first consideration is whether variability reflects the outcome of the movement or coordinative aspects underlying that performance. For example, in research in clinical gait the emphasis is often on variability of stride 
parameters to assess the impacts of aging or disease, with the dominant notion that increases in stride parameter variability represent disease-related impairments. The perspective here is that, although these increases in variability can be signatures of disease processes, this may not be an overall reflection of changes in gait. An early example of this was the reduction in coordination variability in the upper body between pelvis and thorax in newly diagnosed individuals with Parkinson's disease. In contrast, stride parameter variability did not differ from healthy controls. ${ }^{4}$

Secondly, in gaining further insights into the role of variability in functional movements and adaptive behavior, we need to move to experimental manipulations that more directly reflect the relations between the organism and the environment and how these change under different task, organismic and environmental constraints. ${ }^{5}$ Recent research in this area includes examinations of postural-manual interactions ${ }^{6}$ and the modification of intrinsic gait coordination dynamics during reaching and grasping objects. ${ }^{8}$ Although postural variability can serve a functional purpose, in some contexts, such as when performing a concurrent precision demanding manual task, variability may interfere with concurrent task performance. A healthy motor system appears capable of optimizing movement variability (e.g. minimizing postural variability when extraneous fluctuations could interfere with task completion) based on the goals and constraints of concurrently performed tasks, ${ }^{6}$ and consequences of task failure. ${ }^{9}$

Finally, we need to further develop approaches to assess the relationship between functional and nonfunctional variability in the degrees of freedom and performance variables. An important conceptual development in this area has been the uncontrolled manifold (UCM) approach. ${ }^{10}$ In the UCM approach an important differentiation is made between functional variability, identified as those fluctuations in the underlying degrees of freedom that leave the endpoint or controlled variable invariant, and non-functional variability, those fluctuations resulting in performance deviations. This approach has been very successful in creating new insights into the functionality of variability in a wide variety of movement paradigms and is still gaining significant recognition.

Despite these developments much of the clinical research on human posture and gait continues to use increased variability as a key indicator of movement pathology and disability. Future research should focus to a greater degree on the function of movement variability in establishing stable organism-environment relationships and the role of variability in extracting task-relevant information to sustain or change these relationships. To give variability its due place, we argue that as shift in focus is needed from a unidimensional association of high variability with pathology or poor performance to a more multidimensional understanding of the role of variability. This shift is in our view essential for understanding adaptive movement behaviors in health and disease.

\section{REFERENCES}

1. Newell KM, Corcos DM. Variability and motor control. 1993: Human Kinetics Publishers Champaign, IL.

2. Hamill J, van Emmerik RE, Heiderscheit BC, Li L. A dynamical systems approach to lower extremity running injuries. Clin Biomech. 1999;14(5):297-308. 
3. Preatoni E, Hamill J, Harrison AJ, Hayes K, Van Emmerik RE, Wilson C, et al. Movement variability and skills monitoring in sports. Sports Biomech. 2013;12(2):69-92.

4. van Emmerik REA, Wagenaar RC, Winogrodzka A, Wolters EC. Identification of axial rigidity during locomotion in Parkinson disease. Arch Phys Med Rehabil. 1999;80(2):18691.

5. Riccio GE. Information in movement variability about the qualitative dynamics of posture and orientation. In: In KM Newell and DM Corcos (Eds.), Variability and motor control, 1993: p. 317-357. Champaign, IL: Human Kinetics.

6. Haddad JM, Rietdyk S, Claxton LJ, Huber JE. Task-dependent postural control throughout the lifespan. Exerc Sport Sci Rev. 2013;41(2):123-32.

7. van Emmerik REA, van Wegen EE. On the functional aspects of variability in postural control. Exerc Sport Sci Rev. 2002;30(4):177-83.

8. Rinaldi NM, van Emmerik R, Moraes R. Changes in interlimb coordination during walking and grasping task in older adult fallers and non-fallers. Hum Mov Sci. 2017;55:121-137.

9. Simon-Kuhn KL, Haddad JM, Huber JE. Multi-task prioritization during the performance of a postural-manual and communication task. Exp Brain Res. 2019;237(4):927-938.

10. Scholz JP, Schöner G. The uncontrolled manifold concept: identifying control variables for a functional task. Exp Brain Res. 1999;126(3):289-306.

Citation: van Emmerik REA, Hamill J, Haddad JM. (2021). Functional Variability in Movement Coordination and Perception. Brazilian Journal of Motor Behavior, 15(5):278-280.

Editors: Dr Fabio Augusto Barbieri - São Paulo State University (UNESP), Bauru, SP, Brazil; Dr José Angelo Barela São Paulo State University (UNESP), Rio Claro, SP, Brazil; Dr Natalia Madalena Rinaldi - Federal University of Espírito Santo (UFES), Vitória, ES, Brazil.

Section Editors (Current Opinion): Dr Luis Augusto Teixeira - University of São Paulo (USP), São Paulo, SP, Brazil; Dr Tibor Hortobágyi - University of Groningen, The Netherlands; Dr Renato de Moraes - University of São Paulo (USP), Ribeirão Preto, SP, Brazil.

Copyright:@ 2021 van Emmerik, Hamill and Haddad and BJMB. This is an open-access article distributed under the terms of the Creative Commons Attribution-Non Commercial-No Derivatives 4.0 International License which permits unrestricted use, distribution, and reproduction in any medium, provided the original author and source are credited.

Funding: There was no funding for this study.

Competing interests: The authors have declared that no competing interests exist.

DOI: https://doi.org/10.20338/bjmb.v15i5.248 